Manuelle Medizin 2016 ·54:137-138

DOI 10.1007/s00337-016-0144-5

Online publiziert: 25. Mai 2016

(c) Springer-Verlag Berlin Heidelberg 2016

CrossMark

\author{
L. Beyer \\ Ärztehaus Mitte, Jena, Deutschland
}

\title{
Stellen wir uns dem internationalen Vergleich
}

Beiträgen, die die Erfahrungen aus der Praxis aufgreifen und sie mit Ergebnissen aus Studien unter Beachtung wissenschaftlicher und ethischer Standards untermauern, wie Wuttke u. Spittank in ihrem Leserbrief aus Heft 2 [2] fordern.

In diesem Heft finden Sie einen Beitrag von Mizher u. Rüegg zur Problematik der Beinlängendifferenz und deren Folge für die Wirbelsäule und die Umstellung in den Bewegungsprogrammen sowie Vorschläge für die Behandlung. Krocker berichtet zu myofaszialen Ganzkörperspannungsphänomenen. „Herzrhythmusstörungen durch Grenzstrangirritation“ ist ein häufig diskutiertes Thema, das als Befund hier von Hage anhand eines Fallbeispiels postuliert wird.

Niemier kommt nach einem zusammenfassenden Überblick aus dem „Barmer GEK-Report Krankenhaus 2015“ zum Schwerpunkt lumbale Rückenschmerzen zu den Folgerungen: „In der stationären Versorgung zeigt sich eine Zunahme der Kapazitäten, der Behandlungen und der Kosten. Eine durch die Sektoren führende strukturierte Versorgung zeigt sich nicht. Die Nationalen VersorgungsLeitlinien, insbesondere die rasche multimodale interdisziplinäre Diagnostik, werden nicht umgesetzt. Eine Strategie zur Bildung von Subgruppen und damit einer gezielten Therapiesteuerung gibt es nicht."

Eine Entwicklung der dem Behandlungsvorgehen zugrunde liegenden theoretischen Konzepte hat in der manuellen Medizin immer dann stattgefunden, wenn Erkenntnisse aus Grundlagenfächern wie der Biomechanik, Muskelund Neurophysiologie gemeinsam diskutiert und einbezogen wurden. Belegt wird dies in der Historie der manuellen Medizin, wie sie in einem Band nach einem 3-jährigen Forschungsvorhabens am Institut für Geschichte der Medizin der Robert Bosch Stiftung dargelegt wird (s. Rezension in diesem Heft). Der Autor des Buchs hat den Wurzeln sowie der Geschichte der Untersuchung und Behandlung mit der Hand (Chiropraktik) unter medizinischem und gesellschaftlichem Aspekt nachgespürt. Hier finden selbst Eingeweihte der manuellen Medizin eine Vielzahl interessanter, neuer Fakten und Begebenheiten zur Entwicklung von Chiropraktik, Osteopathie und manueller Medizin seit 2 Jahrhunderten mit vielen Nachweisen aus deutsch- und englischsprachigen Quellen.

Unsere Zeitschrift ist das Sprachrohr, über das neue Ergebnisse transportiert und einer öffentlichen Diskussion zugänglich gemacht werden. Dies ist effektiver, wenn die Beiträge durch Listung der Zeitschrift einem größeren Kreis der wissenschaftlichen Gemeinschaft frühzeitig bekannt werden. Um ein Listing von $M a$ nuelle Medizin in PubMed zu erreichen, ist es erforderlich, dass die wissenschaftlichen Beiträge nicht nur gelesen, sondern auch in anderen gelisteten Zeitschriften erwähnt - zitiert - werden. Dies ist nicht von heute auf morgen zu erreichen und erfordert die Mitwirkung eines größeren Kreises aktiver wissenschaftlich publizierender Autoren. Wie diese zu guten Arbeiten für die eigene Zeitschrift aktiviert werden können, wird unter den Herausgebern gerade beraten. Viele Mitglieder des wissenschaftlichen Beirats stehen Institutionen vor, in denen wissenschaftlich aktive Mitarbeiter ein großes Interesse an eigenen Publikationen haben, die national und international zitiert werden. Für das Niveau unserer Zeitschrift wäre es vorteilhaft, wenn sich die Mitwirkenden - und Sie sind dazu aufgefordert rer Praxis veröffentlichen und zur Diskussion stellen. Allerdings mangelt es an 
- mit einer gemeinsamen, konsequenten und nachhaltigen Aktion dafür einsetzen, dass sich der Grad an Zitationen unserer Zeitschrift verbessert. Dies wird nicht zuletzt unseren künftigen Autoren nützen. Wir sind überzeugt davon, dass das wissenschaftliche Niveau unserer Fachzeitschrift dem internationalen Vergleich standhalten kann. Unserem Fach und unserem eigenen Anliegen sind wir es schuldig, dass sich das gesteckte wissenschaftliche Niveau und unser Anspruch in einem angemessenen Impact Factor widerspiegeln.

Vielleicht haben Sie in den Sommerwochen etwas Muße, um über einen eigenen Beitrag für die Manuelle Medizin nachzudenken. Die von der Deutschen Stiftung Manuelle Medizin finanzierte Forschungsberatungsstelle steht Ihnen jederzeit für Beratung und Hilfe zur Verfügung.

Ich wünsche Ihnen eine gute Sommerzeit.

Ihr L. Beyer

\section{Korrespondenzadresse}

\section{Prof. Dr. L. Beyer}

Ärztehaus Mitte

Westbahnhofstr. 2, 07745 Jena, Deutschland lobeyer@t-online.de

Interessenkonflikt. L. Beyer gibt an, dass kein Interessenkonflikt besteht.

\section{Literatur}

1. MacDonald R (2015) Somatic dysfunction - the life of a concept. In: Hutson M, Ward A (Hrsg) Textbook of Musculoskeletal Medicine, 2. Aufl. Oxford University Press, Oxford

2. Wuttke M, Spittank H (2016) Anspruch der Manualmedizin an medizinische Forschung und ethische Standards. Man Medizin 54:119
Transparenz der Daten verbes-
sert Arzt-Patienten-Beziehung

Wenn Patienten vollen Zugriff auf ihre medizinischen Befunde und die Notizen des Arztes zu ihrer Erkrankung erhalten, verbessert dies die Arzt-Patienten-Beziehung erheblich. Dies hat eine Gruppe von Forschern der Universität Witten/Herdecke (UW/H) und der Harvard University (Boston, USA) herausgefunden. Zudem stärkt die erhöhte Transparenz in der Behandlung die Mitarbeit und das Selbstmanagement der Patienten, verbessert das Verständnis der medizinischen Probleme und unterstützt die Selbstfürsorge.

Nach Studienlage versteht etwa die Hälfte der Patienten, die zum Arzt gehen, nicht genau, was gerade besprochen wurde. Durch das OpenNotes-Projekt hat sich das Verständnis für das Besprochene radikal verbessert, so das Team. Dadurch, dass die Patienten alles noch einmal nachlesen und auch die Notizen der Ärzte online einsehen können, haben sie die Möglichkeit, sich noch intensiver mit dem Thema zu beschäftigen, etwas noch einmal nachzulesen oder mit Angehörigen und Bekannten darüber zu sprechen. Am Anfang hatten sich 120.000 Patienten beteiligt, mittlerweile sind schon rund 8 Millionen dabei, berichten die Forscher. In zwei Jahren möchten sie 50 Millionen erreichen.

In der Studie gaben mehr als $77 \%$ der Patienten an, durch OpenNotes mehr Kontrolle über ihre Behandlung zu haben als zuvor. Mehr als 60\% konnten durch das Programm ihre Medikation korrekt oder besser dosieren. Und fast alle Befragten fanden mindestens einmal einen Irrtum oder ein Missverständnis in den Unterlagen, die sie dank der freien Zugänglichkeit schnell korrigieren lassen konnten. Einige Patienten gaben zudem zu, Informationen zum Schutz ihrer Privatsphäre zunächst zurückgehalten zu haben, bis innen durch die Einsicht in die Unterlagen klar geworden sei, dass sie zur Behandlung wichtig seien.

Letztlich ist dieser transparente Ansatz ein Gewinn für beide Seiten, lautet das Resümee: Die Patienten haben mehr Vertrauen, nehmen ihre Medikamente wie verschrie- ben, erinnern sich besser an das Besprochene und arbeiten aktiv an ihrer Gesundung mit. Die Ärzte können dadurch ihre Kommunikation verbessern, haben besser informierte und vorbereitete Patienten und auch andere Ärzte und Pflegende können besser über die gewünschte Behandlung unterrichtet werden. Und zu guter Letzt hilft das auch dem Gesundheitssystem. Wenn besser kommuniziert und die Dosierung der Medikamente eingehalten wird, medizinische Fehler vermieden werden und der Arzt seine Zeit effizienter nutzen kann, reduziert das am Ende auch die Kosten der Behandlung.

Kontakt: Prof. Dr. Tobias Esch, tobias.esch@uni-wh.de oder 02302 / 926-838

Weitere Informationen:

www.opennotes.org

Die Studie finden Sie unter: http://bmjopen.bmj.com/content/6/1/ e010034.full

Quelle:Universität Witten/Herdecke 